\title{
Review of: "On-microscope staging of live cells reveals changes in the dynamics of transcriptional bursting during differentiation"
}

\author{
Kinuko Ohneda ${ }^{1}$ \\ 1 Tohoku University
}

Potential competing interests: The author(s) declared that no potential competing interests exist.

In this manuscript, Jeziorska et al. monitored mouse alpha-globin transcription in live cells during erythroid cell differentiation of mES cells. The authors utilized PP7 tagging of RNA transcripts that has been previously developed in non-mammalian cells, and the cells were grouped into 6 stages of differentiation (DS1-DS6) based on the expression of CD71 and Ter119. Since the FACS sorting affected the cell viability, a live-cell antibody staining was performed for defining erythroblast stages. As summarized in Fig.1, three parameters, burst frequencies (ON duration), burst amplitude, and Fano factor as a mark of variability were analyzed for cells at each differentiation stage. The authors found that most cells displayed low burst amplitude (Basal). The high ON, high amplitude cells that have a low Fano factor(stable) were more frequently observed at stages DS3 and DS4, when alpha-globin nascent transcription was at a high level. The low ON, high amplitude cells that have a high Fano factor (variable) were frequently observed at stages DS1, DS2 and DS5. In these cells, the output of alpha-globin nascent transcription was low. This is the first live-cell study of transcription dynamics during mammalian differentiation. As the authors described, this paper introduces technical advances for imaging of transcription dynamics during differentiation and development and the results are interesting. However, the authors draw a conclusion in combination with previous work (Fig.6) and some interpretations were too speculative. Moreover, I felt that some descriptions in the results section were a little hard to follow.

1. The live cell imaging studies were done by using day 7 EB-derived cells for all differentiation stages. The cells at stages DS1 and DS2 at day 7 of culture may not have the ability to differentiate into mature erythroblasts and have distinct features. The live cell imaging experiments of the cells at stages DS1 and DS2 should be studied at day 5 of EB culture to confirm that the results are consistent with the DS1 and DS2 cells at day 7.

1. It is surprising that a considerable amount of variation in the pattern of transcription was observed at all stages of differentiation, and more than $70 \%$ of the cells displayed basal amplitude even at differentiation stages DS3 and DS4. It would be interesting if the variability of transcription pattern is a characteristic of developmentally regulated gene transcription. In this regard, it would be informative 
the live cell imaging studies of constitutively active genes such as housekeeping genes and silent genes such as neuronal genes.

1. In the introduction section, the authors described that four cell divisions were observed during erythroid cell differentiation. Is the pattern of nascent transcription affected by cell-cycle?

1. Since the PCP-GFP transgene was randomly integrated into the genomes, the fluorescent intensity of the transcription spot might be affected by the activity of integrated site. In the methods section, the authors described that the clones with medium and relatively uniform transgene expression level as assessed by GFP fluorescence were chosen for the analysis. Given that GFP fluorescence is active when alpha-globin gene transcribed and the pattern of transcription was variable, the explanation and/or data should be added for the clone selection.

1. Although the Hba-a1/2 mRNA level was shown at different culture days, it also should be shown in each differentiation stage (F1-F6 cells).

1. Fig.3A: Indices for CD71, PCP-GFP, and Ter119 are hardly visible.

1. Fig.5A: Is this the analysis for all cells at day 7 of EB culture?

1. Fig.5C: The meaning of the "amplitude residual" is difficult to understand and should be explained in the main text. How are the state of cells with amplitude residual below zero?

1. Ext.Data Fig.1: Please add an allow to show colocalization of red and green spot. 